\title{
Stability of ice-sheet grounding lines
}

\author{
By Richard F. Katz ${ }^{1}$ And M. Grae Worster ${ }^{2}$ \\ ${ }^{1}$ Department of Earth Science, University of Oxford, Parks Road, Oxford OX1 3PR, UK. \\ Email: richard.katz@earth.ox.ac.uk \\ ${ }^{2}$ Institute of Theoretical Geophysics, Department of Applied Mathematics and Theoretical \\ Physics, University of Cambridge, Wilberforce Road, Cambridge CB3 0WA, UK.
}

Recent observations of the West Antarctic Ice Sheet document rapid changes in the mass balance of its component glaciers. These observations raise the question of whether changing climatic conditions have triggered a dynamical instability in the ice sheet-ice shelf system. The dynamics of marine ice sheets are sensitive to grounding-line position and variation, characteristics that are poorly captured by most current models. We present a theory for grounding-line dynamics in three spatial dimensions and time. Our theory is based on a balance of forces across the grounding line; it is expressed as a differential equation that is analogous to the canonical Stefan condition. We apply this theory to the question of grounding-line stability under conditions of retrograde bed-slope in a suite of calculations with different basal topography. A subset of these have basal topography inspired by the Pine Island glacier, where basal depth varies in both the along-flow and across-flow directions. Our results indicate that unstable retreat of the grounding line over retrograde beds is a robust feature of models that evolve based on force balance at the grounding line. We conclude, based on our simplified model, that unstable grounding-line recession may already be occurring at the Pine Island glacier.

Keywords: ice sheet, grounding line, ice shelf, Antarctica, Pine Island, glacier, sea level

\section{Introduction}

The glaciers of the West Antarctic Ice Sheet comprise a volume of ice equivalent to a rise in sea level of approximately 3.3 meters (Bamber et al., 2009). This ice sheet is, in large part, grounded on rock that is below sea level. Un-grounding of a relatively small portion of the ice sheet, and the attendant change in mass balance, could result in a significant change in global sea level. Indeed, recent geological observations suggest that sea level rose by 2-3 metres on a human timescale $(\sim 100$ yrs) during the terminal phase of the last interglacial period (Blanchon et al., 2009). Understanding the dynamics of Antarctic grounding lines and how they may respond to climatic perturbations is a critical component in predictions of future environmental change. A particularly important question regards the stability of grounding lines: can gradual modulation in the environmental conditions of Antarctica lead to massive, irreversible grounding line recession and disintegration of the West Antarctica Ice Sheet? Novel theories of grounding line dynamics may help us to answer this question, and to interpret observations of rapid change in West Antarctica.

Recent observations of glaciers that flow into the Amundsen Sea suggest that they are undergoing accelerating change. Thomas et al. (2004) described altimetry measurements that document glacial thinning and estimated that glaciers in this region are discharging almost $60 \%$ more ice per year than they are accumulating. More recently, Wingham et al. (2009) documented rapid changes in ice thickness of the Pine Island glacier. Rignot (2008) used synthetic-aperture interferometry on radar observations collected over a period from 1974 to 2007 to determine changes in flow rates of the Pine Island and Smith glaciers. According to this work, the two glaciers sped up by $42 \%$ and 83\%, respectively, between 1996 and 2007; both also experienced a significant grounding line recession during this period. The measurements show that acceleration of the Smith glacier was substantially larger in 2006-2007 than in previous years. Rignot et al. (2008) estimates that the combined flux of ice across the grounding line of the Pine Island and Thwaites glaciers in 2006 was $85 \pm 26$ gigatonnes per year more than their combined ice accumulation rate at that time; this imbalance is a factor of two larger than measured ten years earlier. In Greenland, geochemical evidence reported by Briner et al. (2009) indicates a rapid deglaciation of the Sam Ford Fjord in the early Holocene, with inferred topographic control of the rate of glacial retreat. 
These and other observations again raise a crucial question (e.g. Weertman, 1976; Bentley, 1997): do recent changes represent a mounting, catastrophic collapse of West Antarctic Ice Sheet associated with unstable retreat of grounding lines (e.g. Weertman, 1974) or, rather, a steady and controlled adjustment to changing climatic forcing (e.g. Van der Veen, 1985). Since the dominant resistance to flow in ice sheets is provided by basal drag (e.g. Van der Veen, 1999), a reduction in the grounded area of an ice sheet would plausibly lead to more rapid flow of ice. Ice sheet buttressing by embayed ice shelves could also contribute a stabilising resistance to flow; disintegration of an ice shelf would relieve this buttressing and allow for flow acceleration (Dupont \& Alley, 2005). While buttressing may be important to the overall dynamics of ice sheets, we neglect it in the current analysis and focus instead on the dynamical changes associated with grounding line migration.

Although other authors had proposed the idea earlier, Weertman (1974) published the first mathematical theory for grounding line instability. The theory is based on analysis of a 2D ice sheet and its junction with a 2D ice shelf; the conditions applied at the junction are the flotation condition and continuity of ice thickness. Weertman (1974) concluded that glaciers grounded below sea-level on beds that slope upward in the flow direction are inherently unstable to grounding line recession (such beds are referred to here as being retrograde). Other authors have developed ice sheet-ice shelf models in which the grounding line position is governed by the flotation condition or its total differential (e.g. Thomas \& Bentley, 1978; Hindmarsh, 1996; Hindmarsh \& LeMeur, 2001; Vieli \& Payne, 2005; Pattyn et al., 2006). Comparative analysis of these models by Vieli \& Payne (2005) suggested that differences in predicted grounding line motion could be attributed to issues with the numerical methods, as well as to differences in the formulation of grounding line motion. This inaccuracy should not come as a surprise: as noted by Schoof (2007a) and Robison et al. (2009), solving for the position of the grounding line is a free-boundary problem (like the canonical Stefan problem) and as such, it requires two independent conditions at the moving boundary. The flotation condition can only be used as one of these.

Chugunov \& Wilchinsky (1996) and Schoof (2007b) applied asymptotic analyses to solve for the stress conditions in a boundary layer that connects the ice sheet to the ice shelf. In this narrow region, shear stresses (that dominate in the sheet) and longitudinal stresses (that dominate in the shelf) are of similar magnitude. Both Chugunov \& Wilchinsky (1996) and Schoof (2007b) showed that by collapsing the width of this narrow transition zone to zero, the boundary layer can be approximated with a boundary condition on the ice sheet. There are several key difference between these two studies, however: Chugunov \& Wilchinsky (1996) applied a no-slip boundary condition at the base of a Newtonian ice sheet and retained the vertical shear term in the horizontal momentum equation while Schoof (2007b) used Glen's flow law, applied a friction condition at the base, and neglected vertical shear.

New work on a Newtonian ice sheet model by Robison et al. (2009) began with the simplifying assumption that the transition zone between the ice sheet and shelf can be collapsed to a boundary of zero thickness. The authors derived a grounding line equation based on the balance of forces between the ice sheet and the ice shelf at this boundary. Steady-state solutions for position of the grounding line were determined for downward sloping (prograde) beds. However, Robison et al. (2009) did not consider the existence or stability of steady solutions for upward sloping beds. Furthermore, none of the authors who have developed the free-boundary approach to deriving a governing equation for the grounding line position have considered a three dimensional ice sheet. It is thus the purpose of the present work to extend the theory of Robison et al. (2009) to 3D and, furthermore, to model ice sheet flow and grounding line position over a bed with topographic variation in both the along-flow and cross-flow directions.

The stability of a grounding line governed by the balance of forces was studied by Schoof $(2007 a)$. His work showed that under the assumptions particular to his model (i.e. rapid sliding, no internal shear), there are no steady-state solutions for a $2 \mathrm{D}$ ice sheet with the grounding line on an upward sloping bed. Work by Nowicki \& Wingham (2008) using numerical solutions of the 2D Stokes equation supports this result but is inconclusive when a no-slip condition is applied at the base of the ice sheet. Like the case investigated by Schoof (2007a), non-slipping ice represents an end-member case that is worth investigating. While our analysis neglects basal sliding and non-Newtonian ice viscosity, it incorporates internal shear and three dimensional effects that are absent from the work of Schoof $(2007 a)$.

In the next section of this paper we provide details of the model derivation, scaling, and numerical solution procedure. The theoretical development in this section is an extension of the ice 
sheet and grounding line model of Robison et al. (2009) from two to three dimensions. In the 2D model, the dynamics of the ice shelf are described by the leading order terms in lubrication theory: the stress is hydrostatic and the strain is purely extensional. For the general case of a 3D ice shelf bounded by a curved grounding line, the dynamics are more complex. In the theory developed below we ignore this additional ice-shelf complexity and adopt the $2 \mathrm{D}$ theory without modification. This is a deficiency in our model and a target for future work.

Having described the theory, we present numerical solutions for various physical scenarios in $\S 3$. In $2 \mathrm{D}$ model runs, we concentrate our attention on the stability of an ice sheet resting on the basal topography proposed by Schoof $(2007 a)$ that contains a continental shelf with an upward bulge (and retrograde slope) near its margin. We present results on $3 \mathrm{D}$ model runs in $\S 3 \mathrm{~b}$ and $\S 3 \mathrm{c}$; the former considers the problem of steady-state grounding line profiles for flow down shallow valleys, while the latter considers the dynamics and stability of grounding line position over more complex basal topography. Section 4 contains a summary of results, plus further calculations relevant to the stability of the Pine Island glacier in West Antarctica. Conclusions are drawn in $\S 5$.

\section{Model derivation}

Following Robison et al. (2009), we consider an ice sheet of density $\rho$ and viscosity $\mu$ flowing into sea-water of density $\rho_{w}$ and negligible viscosity. The ice sheet is grounded on a bed given by the surface $z=-b(x, y, t)$ where $x$ and $y$ are horizontal coordinates, $z$ is a vertical (upwards) coordinate and $t$ is time. The time dependence of $b$ will represent changes in sea level; we do not consider erosion or sedimentation processes, although these may be important (Alley et al., 2007). Figure 1 is a schematic diagram of the domain and coordinate system.

The surface of the ice sheet is given by $z=h(x, y, t)$. In the present work we will be interested only in the dynamics of the ice sheet and hence will neglect the ice shelf, except in considering the force it exerts on the sheet at the grounding line. The grounding line has a position $x=x_{G}(y, t)$ that we require to be a single-valued function of $y$.

\section{(a) The ice sheet}

Force balance in the ice sheet is governed by leading-order terms of the Shallow Ice Equation (e.g. Fowler \& Larson, 1978; Hutter, 1983; Mangeney \& Califano, 1998), giving a pressure within the ice sheet that is hydrostatic, $p=\rho g(h-z)$. Horizontal pressure gradients are balanced by vertical shear according to

$$
\frac{g}{\nu} \nabla_{x} h=\frac{\partial^{2} \mathbf{v}}{\partial z^{2}}
$$

where $\nu=\mu / \rho$ is the kinematic viscosity, $\mathbf{v}=(u, v)$ is the horizontal vector flow velocity, $g$ is the acceleration due to gravity, and $\nabla_{x}$ is the horizontal gradient (the subscript is dropped below, but it is implied unless otherwise noted). In writing (2.1), we have neglected the down-slope component of gravity, which restricts the validity of this equation to gently sloping beds with $|\nabla b| \ll 1$.

We apply no-slip and no-shear-stress boundary conditions at the bottom and top of the ice sheet, respectively,

$$
\begin{array}{rlrl}
\mathbf{v} & =\mathbf{0} & (z=-b), \\
\frac{\partial \mathbf{v}}{\partial z}=\mathbf{0} & (z=h) .
\end{array}
$$

Integrating (2.1) in $z$ twice, subject to these boundary conditions gives

$$
\mathbf{v}=-\frac{g}{2 \nu}(\nabla h)(z+b)[2 H-(z+b)]
$$

where $H=h+b$ is the thickness of the ice sheet. A third integration in $z$ gives the ice flux,

$$
\mathbf{q}=-\frac{g H^{3}}{3 \nu} \nabla h
$$

Using (2.5), the mass balance equation can be written as

$$
\frac{\partial H}{\partial t}=-\nabla_{x} \cdot \mathbf{q}=\frac{g}{3 \nu} \nabla_{x} \cdot\left(H^{3} \nabla h\right),
$$


where $\nabla_{x}$. is the horizontal divergence operator (again, the subscript is dropped below). This equation states that a change in the thickness of the ice sheet at a point in $(x, y)$ is due to the divergence of the horizontal volume flux at that point. In writing 2.6 we have neglected accumulation and ablation of ice, which are clearly important in large-scale models of ice sheets (e.g. Pollard \& Deconto, 2009).

We consider domains that are periodic in the $y$-direction, so equation (2.6) requires two boundary conditions. The first of these specifies a fixed flux at the inflow-boundary,

$$
-\frac{g H^{3}}{3 \nu} \frac{\partial h}{\partial x}=q_{0} \quad(x=0)
$$

while the second is the standard flotation condition at the grounding line,

$$
\rho H=\rho_{w} b \quad\left(x=x_{G}\right) .
$$

As discussed in the introduction, the equations above do not provide enough information to determine the position of the grounding line $x_{G}$. To do so, we require another condition at $x_{G}$; this will take the form of a force balance between the ice sheet and shelf. To obtain the force exerted by the ice sheet at the grounding line we begin by considering stresses within the ice. Using the same arguments as Robison et al. (2009) we obtain the horizontal stresses as

$$
\begin{aligned}
\sigma_{x x} & \equiv-p+2 \mu \frac{\partial u}{\partial x}=-\rho g(h-z)+4 \mu \frac{\partial u}{\partial x}+2 \mu \frac{\partial v}{\partial y}, \\
\sigma_{y y} & \equiv-p+2 \mu \frac{\partial v}{\partial y}=-\rho g(h-z)+4 \mu \frac{\partial v}{\partial y}+2 \mu \frac{\partial u}{\partial x}, \\
\sigma_{x y} & \equiv \mu\left(\frac{\partial u}{\partial y}+\frac{\partial v}{\partial x}\right) .
\end{aligned}
$$

Integrating these stresses over the thickness of the ice sheet and using equation (2.4), we obtain the components of the vertically integrated horizontal stress tensor $\mathbf{N}^{\text {sheet }}$ as

$$
\begin{aligned}
& N_{x x}^{\text {sheet }}=-\frac{1}{2} \rho g H^{2}+2 \mu\left[\frac{\partial q_{x}}{\partial x}+\nabla \cdot \mathbf{q}+\frac{g H^{2}}{2 \nu}\left(2 \frac{\partial h^{2}}{\partial x}+\frac{\partial h^{2}}{\partial y}\right)\right], \\
& N_{y y}^{\text {sheet }}=-\frac{1}{2} \rho g H^{2}+2 \mu\left[\frac{\partial q_{y}}{\partial y}+\nabla \cdot \mathbf{q}+\frac{g H^{2}}{2 \nu}\left(2 \frac{\partial h^{2}}{\partial y}+\frac{\partial h^{2}}{\partial x}\right)\right], \\
& N_{x y}^{\text {sheet }}=\mu\left[\frac{\partial q_{x}}{\partial y}+\frac{\partial q_{y}}{\partial x}+\frac{g H^{2}}{\nu} \frac{\partial h}{\partial x} \frac{\partial h}{\partial y}\right] .
\end{aligned}
$$

\section{(b) The ice shelf}

Although the ice sheet described above has variations in both the $x$ and $y$-directions, for simplicity we adopt the same 2D model of the ice shelf that was proposed by Robison et al. (2009). In this model the ice shelf is floating in local hydrostatic equilibrium; the air above and water below impose no tangential stresses. The horizontal velocity of the ice is taken to be independent of depth. The depth-integrated longitudinal stress (viscous plus cryostatic) in the shelf is balanced by the hydrostatic pressure from the ocean underneath, acting on the sloped lower surface of the shelf. This depth-integrated stress is given by combining equations (2.7) and (2.10) of Robison et al. (2009) as

$$
N_{x x}^{\text {shelf }}=-\frac{1}{2} \frac{\rho^{2} g}{\rho_{w}} H^{2},
$$

and is equal to the depth-integrated hydrostatic pressure of the ocean at the grounding line. We adapt the model by assuming that the calculated stresses are aligned in the direction normal to the edge of the ice shelf, at the grounding line; i.e. we take $N_{n n}^{\text {shelf }} \approx N_{x x}^{\text {shelf }}$. The direction normal to the grounding line is defined below in equation (2.16).

It is important to note here that the use of a $2 \mathrm{D}$ model for the ice shelf is strictly valid only when the grounding line position is independent of $y$. This will not be the case in some of the calculations to follow. The assumption of a 2D shelf is thus a deficiency of our model; addressing this deficiency is a target for future work. 


\section{(c) The grounding line}

The equation governing the position of the grounding line will be determined by balancing stresses between the ice shelf and the ice sheet. The ice sheet normal stress at the grounding line is $N_{n n}^{\text {sheet }}=\mathbf{n} \cdot \mathbf{N}^{\text {sheet }} \cdot \mathbf{n}$, where $\mathbf{n}$ is a unit vector normal to the line $x_{G}(y, t)$ given by

$$
\mathbf{n}=\frac{\mathbf{i}-\partial_{y} x_{G} \mathbf{j}}{\sqrt{1+\left(\partial_{y} x_{G}\right)^{2}}} .
$$

Here, $\partial_{y}$ is a partial derivative in the $y$-direction, and $\mathbf{i}$ and $\mathbf{j}$ are unit vectors in the $x$ and $y$ directions, respectively.

Expanding the ice-sheet normal stress at $x_{G}$ gives

$$
N_{n n}^{\text {sheet }}=-\frac{1}{2} \rho g H^{2}+2 \mu\left(\boldsymbol{\nabla} \cdot \mathbf{q}+\mathbf{n} \cdot \mathbf{J} \cdot \mathbf{n}+\frac{g H^{2}}{2 \nu} \mathbf{n} \cdot \mathbf{H} \cdot \mathbf{n}\right)
$$

where

$$
\mathbf{J}=\left[\begin{array}{ll}
\partial_{x} q_{x} & \partial_{y} q_{x} \\
\partial_{x} q_{y} & \partial_{y} q_{y}
\end{array}\right] \text { and } \mathbf{H}=\left[\begin{array}{cc}
2\left(\partial_{x} h\right)^{2}+\left(\partial_{y} h\right)^{2} & \left(\partial_{x} h\right)\left(\partial_{y} h\right) \\
\left(\partial_{x} h\right)\left(\partial_{y} h\right) & 2\left(\partial_{y} h\right)^{2}+\left(\partial_{x} h\right)^{2}
\end{array}\right]
$$

Assuming a balance between the forces normal to the grounding line from the ice sheet and the ice shelf (see above, $\S 2 \mathrm{~b}$ ) gives

$$
\left[\frac{g^{\prime} H^{2}}{4 \nu}\right]_{\text {shelf }}=\left[\boldsymbol{\nabla} \cdot \mathbf{q}+\mathbf{n} \cdot \mathbf{J} \cdot \mathbf{n}+\frac{g H^{2}}{2 \nu} \mathbf{n} \cdot \mathbf{H} \cdot \mathbf{n}\right]_{\text {sheet }} .
$$

In the limit $\partial / \partial y \rightarrow 0$ this equation reduces to the one-dimensional version,

$$
\left[\frac{g^{\prime} H^{2}}{8 \nu}\right]_{\text {shelf }}=\left[\frac{\partial q}{\partial x}+\frac{g}{2 \nu} H^{2}\left(\frac{\partial h}{\partial x}\right)^{2}\right]_{\text {sheet }},
$$

which appears in Robison et al. (2009) as equation (2.30).

Combining the mass balance equation (2.6) with the time-derivative of the flotation condition (2.8) gives an expression for the flux divergence,

$$
\boldsymbol{\nabla} \cdot \mathbf{q}=\dot{x}_{G}\left(\frac{\partial H}{\partial x}-\rho^{\prime} \frac{\partial b}{\partial x}\right)-\rho^{\prime} \frac{\partial b}{\partial t},
$$

where $\dot{x}_{G}=\partial x_{G} / \partial t$ and we have defined $\rho^{\prime}=\rho_{w} / \rho$. Finally, this can be substituted into (2.19) to give the grounding line equation,

$$
\left[\left(\rho^{\prime}-1\right) \frac{\partial b}{\partial x}-\frac{\partial h}{\partial x}\right] \dot{x}_{G}=\mathbf{n} \cdot \mathbf{J} \cdot \mathbf{n}+\frac{g H^{2}}{2 \nu} \mathbf{n} \cdot\left(\mathbf{H}-\frac{g^{\prime}}{2 g} \mathbf{I}\right) \cdot \mathbf{n}-\rho^{\prime} \frac{\partial b}{\partial t}
$$

at $x=x_{G}(y, t)$. I represents the identity matrix.

\section{(d) Non-dimensionalization and change of coordinates}

We define the following scales

$$
\begin{gathered}
S_{h}=\left(\frac{6 \nu q_{0}}{g}\right)^{1 / 3}\left(\frac{g}{g^{\prime}}\right)^{1 / 6}, \\
S_{x}=2\left(\frac{6 \nu q_{0}}{g}\right)^{1 / 3}\left(\frac{g}{g^{\prime}}\right)^{2 / 3}, \\
S_{t}=\frac{2}{q_{0}}\left(\frac{6 \nu q_{0}}{g}\right)^{2 / 3}\left(\frac{g}{g^{\prime}}\right)^{5 / 6}
\end{gathered}
$$


where $q_{0}$ is a constant flux with dimensions of area per time. These scales are used to nondimensionalize variables

$$
(\hat{h}, \hat{H}, \hat{b})=(h, H, b) / S_{h}, \quad(\hat{x}, \hat{y})=(x, y) / S_{x}, \quad \hat{t}=t / S_{t},
$$

and $\hat{\mathbf{q}}=\mathbf{q} / q_{0}$. In terms of dimensionless variables, the grounding line equation becomes

$$
\left[\left(\rho^{\prime}-1\right) \frac{\partial \hat{b}}{\partial \hat{x}}-\frac{\partial \hat{h}}{\partial \hat{x}}\right] \dot{\hat{x}}_{G}=\mathbf{n} \cdot \hat{\mathbf{J}} \cdot \mathbf{n}+3 \hat{H}_{G}^{2} \mathbf{n} \cdot\left(\frac{\hat{\mathbf{H}}}{2}-\mathbf{I}\right) \cdot \mathbf{n}-\rho^{\prime} \frac{\partial \hat{b}}{\partial \hat{t}},
$$

where $\hat{H}_{G}(\hat{y}, \hat{t})=\rho^{\prime} \hat{b}\left(\hat{x}_{G}, \hat{y}, \hat{t}\right)$ is the ice thickness at the grounding line. Below, we drop hats from dimensionless variables.

To facilitate discretization of the problem on a regular grid we map the domain to a rectangular one using the transformation

$$
(x, y, t) \rightarrow\left(\xi=\frac{x}{x_{G}}, y, t\right),
$$

where $\xi$ goes from zero at $x=0$ to one at $x=x_{G}$. With this change,

$$
\begin{aligned}
\frac{\partial}{\partial x} & \rightarrow \frac{\partial \xi}{\partial x} \frac{\partial}{\partial \xi}=\frac{1}{x_{G}} \frac{\partial}{\partial \xi} \\
\frac{\partial}{\partial y} & \rightarrow \frac{\partial}{\partial y}+\frac{\partial \xi}{\partial y} \frac{\partial}{\partial \xi}=\frac{\partial}{\partial y}-\frac{\xi}{x_{G}} x_{G}^{\prime} \frac{\partial}{\partial \xi} \\
\frac{\partial}{\partial t} & \rightarrow \frac{\partial}{\partial t}+\frac{\partial \xi}{\partial t} \frac{\partial}{\partial \xi}=\frac{\partial}{\partial t}-\frac{\xi}{x_{G}} \dot{x}_{G} \frac{\partial}{\partial \xi}
\end{aligned}
$$

where $x_{G}^{\prime}$ is the $y$-derivative of the $x$-position of the grounding line.

With dimensionless variables and transformed coordinates the mass balance equation (2.6) becomes

$$
\frac{\partial H}{\partial t}-\frac{\xi}{x_{G}} \dot{x}_{G} \frac{\partial H}{\partial \xi}=\nabla_{\xi} \cdot H^{3} \nabla_{\xi} h
$$

where

$$
\nabla_{\xi}=\frac{1}{x_{G}} \frac{\partial}{\partial \xi} \mathbf{i}+\left(\frac{\partial}{\partial y}-\frac{\xi}{x_{G}} x_{G}^{\prime} \frac{\partial}{\partial \xi}\right) \mathbf{j}
$$

The boundary conditions (2.7) and (2.8) become

$$
\begin{aligned}
\frac{H^{3}}{x_{G}} \frac{\partial h}{\partial \xi}=-1 & (\xi=0), \\
h=\left(\rho^{\prime}-1\right) b\left(x_{G}, y, t\right) & (\xi=1) .
\end{aligned}
$$

The grounding line equation (2.27) was transformed similarly in the numerical code.

\section{(e) Numerical solution}

We discretize the governing equations with implicit time-stepping and a flux-conservative, finite volume scheme. The mass balance equation (2.32) is discretized onto a Cartesian $2 \mathrm{D}$ grid with $n_{i}$ points in the $\xi$-direction and $n_{j}$ points in the $y$-direction; the grounding line equation is discretized onto a $1 \mathrm{D}$ grid with $n_{j}$ points. This yields a system of $\left(n_{i}+1\right) \times n_{j}$ nonlinear algebraic equations in $\left(n_{i}+1\right) \times n_{j}$ variables. Experience has shown us that a solution strategy based on iterative, separate updates of the grounding line position and the ice-sheet height at each time-step is numerically unstable. To avoid this problem we invert the entire, coupled system for $\left\{h_{i j}, x_{G j}\right\}$ at each time-step. This inversion is accomplished using an Incomplete-LU-preconditioned NewtonKrylov (GMRES) method provided by the Portable, Extensible Toolkit for Scientific Computation (PETSc, Balay et al., 2001, 2004).

The calculation are initialised with an asymptotic solution to the $2 \mathrm{D}$ governing equations at $t \ll 1$ (Robison et al., 2009). For 2D calculations (e.g. fig. 2a), we use 2000 grid cells, evenly distributed in the $\xi$-direction. For 3D calculations, unless noted in the figure caption, we use a grid of $120 \times 120$ cells, evenly distributed in $\xi, y$; the grounding line position is discretized over the same 
number of cells as in the $y$-direction of the ice sheet. The time-step size is adjusted at each step to give a maximum proportional change in the grounding line position of approximately $0.05 \%$. We accept the solution at any time-step when the L2 norm of the non-linear system residual is less than $10^{-8}$. The code has been verified in the simple case of a $2 \mathrm{D}$ ice sheet with constant bed-slope by comparison with an analytical solution from Robison et al. (2009). This work demonstrated that the theory admits a single solution for the grounding line position on any contiguous section of prograde bed. As a qualitative check for the case of more complex bed topography, we compare results with the model of Schoof $(2007 a)$ in the next section.

\section{Results}

\section{(a) Stability in $2 D$ models}

It is instructive to consider grounding line instability in comparison to the results of Schoof $(2007 a)$. To do so, we adopt the profile of basal topography proposed in equation (10) of that paper (shown in our fig. 2a). This basal topography profile mimics the form of many of the icesheet beds around Western Antarctica: a long, shallow, prograde continental slope curves upward into a shallower outer rise prior to dropping sharply into the deep ocean. The presence of retrograde slopes on the upstream side of this outer rise creates a region where grounding lines are expected to be unstable. Our results affirm this expectation.

Figure 2a shows equilibrium profiles of ice-sheet surface height at various sea-level heights. Each curve was calculated by stepping sea level in increments $(\sim 1$ metre $)$ from the previous value and allowing the height and grounding-line position to evolve according to the governing equations until a new steady-state was reached. The critical step over which unstable grounding line recession occurred was refined to $0.4 \mathrm{~mm}$ change in sea level.

Like Schoof $(2007 a)$, our model produces a hysteresis loop, shown in figure $2 \mathrm{~b}$, for grounding line position as a function of sea-level height. With the grounding line on the outer prograde slope and a rising sea level, the grounding line recedes in small steps, proportional to the change in sea level. When it reaches the crest of the outer topographic rise, an incremental increase in sea level leads to a large leap backwards in grounding line position. The grounding line settles on the inner prograde slope at a position where the basal depth is approximately equal to that of the crest of the outer rise. Further small increases in sea level lead to proportional recession of the grounding line up the inner slope. As sea level is dropped in small steps, a reversal of this process occurs, except that the unstable leap in grounding line position occurs when the grounding line has reached the trough between the inner slope and the outer rise. There, the grounding line leaps forward to the prograde outer slope.

Figure 2c demonstrates that in calculations with different values of the input flux $q_{0}$, the unstable grounding-line transitions occur at different values of sea level. A larger flux of ice results in a thicker ice sheet and therefore requires higher sea level for both prograde and retrograde transitions of the grounding line position. More importantly, for fixed sea level, a transition in grounding line position can result from a change in the ice flux. Although our model does not explicitly include basal melting of ice at the grounding line as does Walker et al. (2008), this melting may be similar in effect to a reduction in flux $q_{0}$. As figure $2 \mathrm{c}$ shows, such a reduction can lead to unstable grounding line retreat.

To investigate the dynamics of a retrograde transition event, we consider a fixed flux $q_{0}$ and begin the calculation with a steady-state ice sheet profile that is on the verge of a transition. A step-increase in sea level is applied and the system is allowed to evolve according to the governing equations. Figure 3 shows the results of an example calculation with $q_{0}=0.02$ (other parameters as in fig. 2a). In this case, the step in sea level triggered a retrograde transition that shifted the grounding line $x_{G}$ by almost $500 \mathrm{~km}$. Figure 3 a shows the grounding line position and the ratio of the flux across the grounding line to that entering the domain $q_{G} / q_{0}$, as a function of time, from the time of the change in sea level. The first phase of the transition is slow due to the shallow retrograde slopes at the crest of the outer rise. As the grounding line reaches the steeper retrograde slope of the outer rise, the transition enters the second phase. In this phase, the basal depth at the grounding line increases, leading to an accelerating change in ice flux across the grounding line and a thinning of the ice sheet that, in turn, causes further grounding line recession. Figure 3b shows that $q_{G} / q_{0}$ reaches a maximum when the grounding line is at the deepest point between the 
inner prograde slope and the outer rise. In phase three of the transition, the grounding line climbs the inner slope. As the basal depth at the grounding line decreases, so too does the flux ratio; the transition ceases as the flux ratio approaches unity.

For the basal topographic profile adopted from (Schoof, 2007a), the rapid phases (2 and 3) of the transition in grounding line position occur over about 10 thousand years, a period that is nearly independent of the influx $q_{0}$ and the step-size in sea level, for values considered. The maximum flux ratio during the transition decreases by about $10 \%$ with changing background flux $q_{0}$, from 1.57 at $q_{0}=0.02$ to 1.42 at $q_{0}=0.045$. These results are specific to the basal profile proposed by Schoof (2007a) and used here (as well as the chosen model and parameter values). Below, in $\S 4 \mathrm{~b}$, we demonstrate that a different profile of basal topography yields a grounding-line transition that is significantly more rapid. Beyond this dependence, the dynamical timescale associated with unstable grounding line recession will be affected by the rheology of the ice and the basal conditions (Joughin et al., 2009). We expect that our use of Newtonian rheology and a no-slip condition at the base of the grounded ice yields an overestimate of the dynamical timescale for recession.

\section{(b) Steady-states in $3 D$}

The governing equations derived above permit the calculation of grounding line evolution in three dimensions. With the addition of the across-flow $(y)$ direction, we have the opportunity to specify variations in basal topography in both $x$ and $y$. The calculations presented in this section use a particularly simple basal topography: a constant, prograde slope that is modulated by a sinusoidal function in the $y$-direction

$$
b(x, y)=x[\alpha-\beta \cos (k y)] .
$$

Here $\alpha$ is the mean slope in the $x$-direction, $\beta$ is the amplitude of modulation in slope, and $k$ is the wavenumber of that modulation in the $y$-direction; variables in (3.1) are dimensional. The ratio $B=\beta / \alpha$ gives the proportional variation of basal depth at fixed $x$ relative to the mean depth at that distance. Our assumption that the downslope component of gravity is negligible (see above) requires that we consider only $\alpha \ll 1$, which is an acceptable restriction because this is uniformly true for natural ice sheets.

Figure 4 shows an example calculation with mean slope $\alpha=3.3 \times 10^{-3}$, giving about 3 metres of basal depth change per kilometre in the $x$-direction, and $\beta / \alpha=0.8$, giving up to $80 \%$ deviation in basal depth at any value of $x$. Setting $k=2 \pi / y_{\max }$ gives a single valley with a width equal to the width of the domain. Although this bed form does not correspond with a specific natural ice sheet, it is reminiscent of the Pine Island glacier, where basal topography confines much of the flow to a broad ice stream underlain by a basal valley (e.g. Vaughan et al., 2006).

Figure 5a shows the characteristics of the ice flux at the grounding line for the calculation depicted in figure 4. The grounding line is curved, but it does not follow a depth contour (fig. 4b). Hence the basal depth at the grounding line varies substantially, although less than it would if $x_{G}$ were a constant, independent of $y$. Given this variation in basal depth, it is not surprising to find that the ice flux across the grounding line is concentrated into the centre of valley. Figure 5a shows that for this value of $\beta / \alpha$, there is almost zero flux at the edges of the domain, along the ridges that flank the valley (recall that the domain is periodic in the $y$-direction).

The components of the ice flux at the grounding line are plotted in figure 5b. Because we expect a strong variation in the flux with $H_{G}$ (equation (2.5)), the curves are scaled by the cube of the ice thickness there and then normalised. The heavy curve representing $\mathbf{q} \cdot \mathbf{n} / H_{G}^{3}$ has little variation across the centre of the grounding line, between $y \approx 5$ and $y \approx 45 \mathrm{~km}$, whereas outside of this region it decays sharply toward zero. This indicates that focusing of flow redistributes ice toward the centre of the valley, and that the flow along the ridge crests is anomalously sluggish relative to the thickness of ice there. Unsurprisingly, the flow has a three-dimensional aspect that is not negligible.

To explore the sensitivity of 3D models to basal topography, we have performed a suite of calculations for different values of the mean bed slope $\alpha$ and the sinusoidal perturbation to the bed slope $\beta$; these are summarised in figure 6 . Panel (a) of this figure shows how the mean grounding line position (averaged over the $y$-direction) depends on the mean bed slope. There is a linear relationship between the inverse slope $1 / \alpha$ and the mean grounding line position. This relation was predicted for the two-dimensional case $(\beta=0)$ by Robison et al. (2009). Our results demonstrate 
that the mean grounding line position is somewhat sensitive to the perturbation in bed slope; larger perturbations yield smaller mean values of $x_{G}$. Figure $6 \mathrm{~b}$ shows the difference between the largest and smallest value of $x_{G}$ as a function of the relative perturbation in bed slope. As expected, this difference grows with increasing $\beta / \alpha$. It is largest for the most steeply sloping bed, where a given amount of relative perturbation translates to the largest amount of actual bed-depth variation at a fixed distance $x$. Finally, figure $6 \mathrm{c}$ shows how the distribution of flux across the grounding line varies with increasing bed-slope perturbation. As shown in figure 4c and 5a, ice flux concentrates above the valley. With increasing $\beta / \alpha$, the ratio of the largest flux to the smallest flux increases, reflecting increased focusing of ice flux toward the centre-line in $y$ where the bed is deepest. For the largest value of $\alpha$, the grounding line is too close to the constant-influx boundary for efficient focusing to occur.

\section{(c) Stability in 3D models}

In the previous sections we examined unstable behaviour of $2 \mathrm{D}$ ice sheets with oscillatory topography (i.e. an outer rise), and stable behaviour of 3D ice sheets flowing over topography that is uniformly prograde in the $x$-direction. Next we consider a $3 \mathrm{D}$ scenario with basal topography that is a combination of these two: it is an internal valley, bounded in the $y$-direction and in the $x$-direction, that is superposed on a gentle, monotonic slope in $x$ (colour contours in fig. 7 ). In this case there is a competition between the monotonic prograde slope, which would yield stable grounding line recession, and the internal valley bounded by a retrograde slope, which would yield unstable grounding line recession. Such a configuration is a highly idealised version of the basal topography beneath the Pine Island glacier (Vaughan et al., 2006) and is hence of special relevance here. Its behaviour cannot be deduced from the calculations described above.

We parameterise the above configuration with the dimensional function

$$
b(x, y)=(\alpha x-729)[1-G(y, \sigma)]-T_{S}(x) G(y, \sigma)
$$

where $x, y$, and $b$ are measured in metres, $G(y, \sigma)$ is a Gaussian centred at the domain's mid-point in $y$ with unit amplitude and standard deviation $\sigma$, and $T_{S}$ is the topographic profile proposed by Schoof $(2007 a)$ and used above in $§ 3$ a.

Figure 7 shows results from two calculations of steady-state grounding line position for a range of sea-level values. With rising sea level, the grounding line retreats toward the left in each panel of the figure. The basal topography in these two calculations differs only in the width of the central valley, given by the standard deviation $\sigma$ of the Gaussian in equation (3.2). The difference in behaviour of the grounding line is striking, however. In the case of the narrower central valley in 7a, there are steady, stable grounding line positions throughout the range in $x$ between the valley's trough and outer rise. In contrast, $7 \mathrm{~b}$ shows that for a wider valley, the grounding line undergoes unstable recession through this same range in $x$. This is the same instability as described in $\S 3$ a but now mediated by three-dimensional flow.

A set of calculations similar to (and including) those shown in figure 7 are summarised in figure 8. Here the grounding line position has been averaged in the $y$-direction and plotted on the $x$-axis. The independent variable, sea level, is plotted on the $y$-axis. With increasing time the curves trace a trajectory that goes from the right side of the figure to the left. Only the grey curves $(\alpha=0.52$ $\mathrm{m} / \mathrm{km})$ with narrow central valleys $(\sigma=5$ and $\sigma=10)$ show stable grounding line recession. A narrower valley drains the grounded ice less efficiently as sea level rises, reducing its destabilising effect. Similarly, for larger $\alpha$, grounded ice flows more rapidly on the monotonic slope outside the central valley. This diminishes the relative contribution of drainage through the valley and hence diminishes its destabilising effect on the grounding line.

\section{Discussion}

\section{(a) Review of the new model}

In the foregoing sections we describe a model of ice-sheet flow and grounding line dynamics extended from that of Robison et al. (2009). Our model differs from that of Schoof $(2007 a)$ in that we neglect basal sliding of the glacier, assume a constant viscosity for the ice, and account for vertical shear stresses. We collapse the transition zone between grounded and ungrounded ice 
proposed by Chugunov \& Wilchinsky (1996) and Schoof (2007b) to a single interface on which normal forces between the ice sheet and the ice shelf are assumed to be in balance.

The results presented above support the theory of unstable recession of ice sheets grounded on retrograde beds (Weertman, 1974). In this paper our focus is on the effect of changes in sea level but, in our model, changes in the influx of ice can also trigger instability. Such flux changes may be qualitatively interpreted as changes in the accumulation rate of ice on the glacier surface, or as changes in the ablation rate of ice at or upstream from the grounding line; both of these have been documented on the West Antarctic Ice Sheet (Rignot et al., 2008).

Our predictions of unstable grounding line retreat are similar to the findings of Schoof $(2007 a)$. These results reinforce the conclusion that for ice sheet/shelf models that enforce boundary conditions at the grounding line, the position of this line should be modelled as a free boundary, and it should be determined with a dynamic boundary condition (e.g. eqn. (2.22)) similar to that used in Stefan-type solidification problems. In contrast, models that solve the full Stokes equation without vertical integration, throughout a unified domain comprised of the ice sheet and shelf, do not require boundary conditions at the grounding line; in that case, the conclusion above is not applicable. These latter type of models could be used as an independent test of our results and conclusions. Below we discuss the implications of our model for understanding the current behaviour of the Pine Island glacier.

\section{(b) Stability of Pine Island glacier}

The theory and calculations described above are idealised in order to highlight the fundamental behaviour of the grounding line; we assumed Newtonian viscosity, one dimensional ice-shelf flow, and smoothed basal topography. With these assumptions, the model is not well-suited to a detailed study of the dynamics of the Antarctic ice cap as a whole, or that of its component glaciers. However, it is interesting to consider calculations that incorporate a basal topography map inspired by observations of the Pine Island glacier. These observations are available thanks to the AGASEA project (Vaughan et al., 2006).

Figure 9a shows a map of the basal topography beneath and around the Pine Island glacier. A transect along the approximate flow-axis of the glacier is given in figure 9b. A cubic polynomial was fit to the transect using least-squares and became the basis for $2 \mathrm{D}$ calculations of grounding line motion described below (details are given in caption of fig. 9). To constrain the influx parameter $q_{0}$ we take the estimate of Rignot (2008) for the average rate of ice input into the Pine Island glacier over the years 1980-2004 of $61 \pm 9$ gigatonnes per year; assuming a grounding line length of $50 \mathrm{~km}$ and an ice density of $900 \mathrm{~kg} / \mathrm{m}^{3}$, this gives an average flux of ice across the grounding line of $q_{0} \approx 0.04 \mathrm{~m}^{2} / \mathrm{sec}$. As above, we assume a Newtonian ice viscosity of $\mu=1 \times 10^{13} \mathrm{~Pa}$-sec. To generate an initial condition for instability calculations, we adjusted sea-level until the calculated steady-state grounding-line position was located just downstream of the crest of the outer rise ( $\sim 360 \mathrm{~km}$ in fig. 9b). A different initial condition was determined for each relevant value of $q_{0}$. The instability calculations were then initialised using these steady-state solutions, but with sea-level raised by several centimetres from the value used to generate the initial condition.

Output from instability calculations is shown in figure 10. In that figure we compare the predicted flux ratio $q_{G} / q_{0}$ as a function of time with data for the Pine Island glacier (the data is derived from table 1 of Rignot (2008)). The data points form an array that bends sharply upward, mirroring the behaviour of the curves. The curves, however, are a poor fit to slope of the data array. This may indicate that the Pine Island grounding line is presently undergoing unstable recession, but at a rate that is much more rapid than our simple models predict. This discrepancy may be due to our modelling assumptions (e.g. Newtonian viscosity, no basal slip), or may be due to a difference in the forcing that is causing recession. For the model curves, the forcing is a small step in sea-level that triggers a change from one stable state to another. Over the time-range of the data series from Rignot (2008), 30 years, the global mean change in sea level was $\sim 6 \mathrm{~cm}$ (Solomon et al., 2007). It is possible that the system crossed a threshold that triggered instability at some sea-level point within this range. It is more likely, however, that the natural system is being forced by a larger single perturbation such as the change in sea level since the last glacial maximum, or, by a set of perturbations including reduced ice accumulation, increased ablation at the grounding line, more basal melt-water, warmer ice, and sea level change. The comparison between data and 
model indicates that the current rate of recession of the Pine Island grounding line is well above what might be considered a minimum estimate from our models.

Given the complex, three dimensional nature of the real Pine Island glacier, with its convergent feeder streams and sub-glacial hydrology, it should be clear that the above model is a very crude representation of reality. Our current model implementation is incapable of capturing this complexity, although in $\S 3 \mathrm{c}$ we presented results for a basal topography inspired by that of the Pine Island glacier. Figure 10 demonstrates that the Pine Island basal profile yields an instability in 2D models that is qualitatively identical to, but more rapid than, that associated with the basal topographic profile introduced by Schoof $(2007 a)$. Incorporation of this profile into a 3D model would mean modification of the basal profile of the central valley, $T_{s}(x)$ in equation (3.2). Based on the basal topography shown in figure 9a, this model would use $\sigma \approx 20 \mathrm{~km}$ and $\alpha<0.17 \mathrm{~m} / \mathrm{km}$. Judging from the behaviour of curves in figure 8, a model with these parameter values would produce a result similar to those in figure 7b: unstable grounding line recession. However, due to the complexity of the natural Pine Island glacier, these results would not provide additional insight over the 2D models. In fact, similar patterns between data and models (fig. 10) suggest that our simple 2D models may capture an essential aspect of the Pine Island glacier.

\section{Conclusions}

The theory developed in $\S 2$ above can be used to describe the dynamics of ice sheets and their grounding lines in three dimensions. We have applied this theory to develop models of grounding line stability and instability in two and three dimensions. In particular, inspired by the questions posed by early investigators (e.g. Weertman, 1974; Thomas \& Bentley, 1978) and by recent observations (Blanchon et al., 2009), we have considered the instability of marine ice sheets grounded on a bed with an outer rise separating the interior of the ice sheet's bed from the steep continental slope. As a benchmark of our model, we have considered a basal profile adopted from Schoof (2007a) and calculated the response of an idealised ice sheet to changes in sea level. Our results are similar to those of Schoof (2007a) and again confirm the hypothesis of unstable grounding line recession over retrograde beds (Weertman, 1974).

We have also examined the stable shape of grounding lines for 3D ice sheets with bed topography that is variable in two dimensions. The results of this work show that ice sheet dynamics play a significant role in determining the position and shape of the grounding line, and the ice flux across it; these features are not simply related to the map of basal elevation.

Three dimensional models were then extended to a case in which an internal valley with a retrograde outer rise is superposed on a prograde bed of constant slope. These calculations showed that the entire ice sheet can be destabilised by the presence of the internal valley, even if it is narrow with respect to the width of the domain. These results bear on sections of an ice sheet affected by strong variations in basal topography such as the Pine Island glacier.

Calculations of grounding line recession over a profile of basal topography derived from AGASEA data (Vaughan et al., 2006) were considered in order to compare the ratio of grounding-line ice flux to accumulation or influx of ice. Data from Rignot (2008) allowed us to compare model results with observed behaviour of the Pine Island glacier. The data follow a trajectory with a rate of change that is larger than model curves. If the Pine Island glacier is indeed undergoing unstable grounding-line recession, the discrepancy between model and data may be due to two key factors that will affect the dynamical adjustment time-scale in our model: our use of Newtonian viscosity, and our application of zero basal sliding. The latter, especially, is known to be important for the dynamics at Pine Island (Joughin et al., 2009).

These results reinforce the conclusion that grounding line dynamics are a critical component of dynamical models of the West Antarctic Ice Sheet. While most continental-scale models of ice sheets still use kinematic (and potentially inconsistent (Vieli \& Payne, 2005)) boundary conditions at the grounding line, the current results indicate that the grounding line should be treated as a free boundary, analogous to the solidification front in a Stefan problem.

The results also underscore the importance of continued research on ice-sheet dynamics. This research should include the development of models that incorporate the detailed bedform and rheology of the West Antarctic Ice Sheet such as Pollard \& Deconto (2009). It should also include the development of idealised models, such as those presented here, that explore the consequences 
of model formulation and assumptions, and probe the basic physics of ice sheets. In particular, more work is needed to predict the timescale of unstable grounding-line retreat.

Finally, our results suggest that in contrast to earlier assessments (e.g. Van der Veen, 1985; Vaughan \& Spouge, 2002), the scenario of unstable grounding line recession on retrograde beds in West Antarctica is likely. Indeed, in the case of the Pine Island glacier, it may be presently occurring.

R. Katz is grateful to R. Hindmarsh and R. Peltier for discussions and encouragement, to O. Sergienko for comments on an early version of the manuscript, to B. Smith and the PETSc team at Argonne National Laboratory for PETSc support and enhancements, and to B. Raup and J. Bohlander from the NSIDC for help with Antarctic grounding line data. The authors acknowledge helpful reviews by R. Gladstone and an anonymous reviewer. Katz received support for this work under award 0602101 from the US National Science Foundation International Research Fellowship Program, and as an Academic Fellow of the Research Councils UK.

\section{References}

Alley, R., Anandakrishnan, S., Dupont, T., Parizek, B. \& Pollard, D. 2007 Effect of sedimentation on ice-sheet grounding-line stability. Science, 315(5820), 1838-1841. (doi:10.1126/science. 1138396)

Balay, S., Buschelman, K., Gropp, W., Kaushik, D., Knepley, M., McInnes, L., Smith, B. \& Zhang, H. 2001 PETSc Web page. Http://www.mcs.anl.gov/petsc.

Balay, S., Buschelman, K., Gropp, W., Kaushik, D., Knepley, M., McInnes, L., Smith, B. \& Zhang, H. 2004 PETSc users manual. Tech. Rep. ANL-95/11 - Revision 2.1.5, Argonne National Laboratory.

Bamber, J., Riva, R., Vermeersen, B. \& LeBrocq, A. 2009 Reassessment of the potential sealevel rise from a collapse of the West Antarctic Ice Sheet. Science, 324(5929), 901-903. (doi: 10.1126/science.1169335)

Bentley, C. 1997 Rapid sea-level rise soon from West Antarctic ice sheet collapse? Science, 275(5303), 1077-1078.

Blanchon, P., Eisenhauer, A., Fietzke, J. \& Liebetrau, V. 2009 Rapid sea-level rise and reef backstepping at the close of the last interglacial highstand. Nature, 458(7240), 881. (doi:10.1038/ nature07933)

Briner, J., Bini, A. \& Anderson, R. 2009 Rapid early Holocene retreat of a Laurentide outlet glacier through an Arctic fjord. Nature Geoscience, 2(7), 496-499. (doi:10.1038/ngeo556)

Chugunov, V. \& Wilchinsky, A. 1996 Modelling of marine glacier and ice-sheet-ice-shelf transition zone based on asymptotic analysis. Ann. Glaciol., 23, 59-67.

Dupont, T. \& Alley, R. 2005 Assessment of the importance of ice-shelf buttressing to ice-sheet flow. Geophys. Res. Letts., 32(4), L04 503. (doi:10.1029/2004GL022024)

Fowler, A. \& Larson, D. 1978 Flow of polythermal glaciers. 1. model and preliminary-analysis. 363(1713), 217-242.

Haran, T., Bohlander, J., Scambos, T. \& Fahnestock, M. 2005 MODIS modaic of Antarctica (MOA) image map. Tech. rep., National Snow and Ice Data Center, Boulder, CO. Http://nsidc.org/data/moa/index.html.

Hindmarsh, R. 1996 Stability of ice rises and uncoupled marine ice sheets. Ann. Glaciol., 23, 94-104.

Hindmarsh, R. \& LeMeur, E. 2001 Dynamical processes involved in the retreat of marine ice sheets. J. Glaciol., 47, 271-282.

Hutter, K. 1983 Theoretical glaciology. Norwell, Mass.: Kluwer Acad. 
Joughin, I., Tulaczyk, S., Bamber, J., Blankenship, D., Holt, J., Scambos, T. \& Vaughan, D. 2009 Basal conditions for pine island and thwaites glaciers, west antarctica, determined using satellite and airborne data. J. Glaciol., 55(190), 245-257. (doi:10.3189/002214309788608705)

Mangeney, A. \& Califano, F. 1998 The shallow ice approximation for anisotropic ice: Formulation and limits. J. Geophys. Res., 103(B1), 691-705.

Nowicki, S. \& Wingham, D. 2008 Conditions for a steady ice sheet-ice shelf junction. Earth Plan. Sci. Lett., 265(1-2), 246-255. (doi:10.1016/j.epsi.2007.10.018)

Pattyn, F., Huyghe, A., Brabander, S. D. \& Smedt, B. D. 2006 Role of transition zones in marine ice sheet dynamics. J. Geophys. Res., 111(F2), F02 004. (doi:10.1029/2005JF000394)

Pollard, D. \& Deconto, R. 2009 Modelling West Antarctic ice sheet growth and collapse through the past five million years. Nature, 458(7236), 329. (doi:10.1038/nature07809)

Rignot, E. 2008 Changes in West Antarctic ice stream dynamics observed with ALOS PALSAR data. Geophys. Res. Letts., 35(12), L12 505. (doi:10.1029/2008GL033365)

Rignot, E., Bamber, J., Van den Broeke, M., Davis, C., Li, Y., Van de Berg, W. \& Van Meijgaard, E. 2008 Recent Antarctic ice mass loss from radar interferometry and regional climate modelling. Nature Geoscience, 1(2), 106. (doi:10.1038/ngeo102)

Robison, R., Huppert, H. \& Worster, M. 2009 Dynamics of viscous grounding lines. J. Fluid Mech. In press.

Schoof, C. $2007 a$ Ice sheet grounding line dynamics: Steady states, stability, and hysteresis. J. Geophys. Res., 112(F3). (doi:10.1029/2006JF000664)

Schoof, C. $2007 b$ Marine ice-sheet dynamics. Part 1. The case of rapid sliding. J. Fluid Mech., 573, 27-55. (doi:10.1017/S0022112006003570)

Solomon, S., Qin, D., Manning, M., Chen, Z., Marquis, M., Averyt, K., Tignor, M. \& Miller, H. (eds) 2007 Contribution of working group $i$ to the fourth assessment report of the intergovernmental panel on climate change. Cambridge University Press.

Thomas, R. \& Bentley, C. 1978 Model for Holocene retreat of the West Antarctic Ice Sheet. Quaternary Res, 10(2), 150-170.

Thomas, R., Rignot, E., Casassa, G., Kanagaratnam, P., Acuna, C., Akins, T., Brecher, H., Frederick, E., Gogineni, P. et al. 2004 Accelerated sea-level rise from West Antarctica. Science, 306(5694), 255. (doi:10.1126/science.1099650)

Van der Veen, C. 1985 Response of a marine ice-sheet to changes at the grounding line. Quaternary Res, 24(3), 257-267.

Van der Veen, C. 1999 Fundamentals of glacier dynamics. A.A. Balkema.

Vaughan, D., Corr, H., Ferraccioli, F., Frearson, N., O’hare, A., Mach, D., Holt, J., Blankenship, D., Morse, D. et al. 2006 New boundary conditions for the West Antarctic ice sheet: Subglacial topography beneath Pine Island Glacier. Geophys. Res. Letts., 33(9). (doi:10.1029/2005GL025588)

Vaughan, D. \& Spouge, J. 2002 Risk estimation of collapse of the West Antarctic Ice Sheet. Climatic Change, 52(1/2), 65-91. (doi:10.1023/A:1013038920600)

Vieli, A. \& Payne, A. 2005 Assessing the ability of numerical ice sheet models to simulate grounding line migration. J. Geophys. Res., 110(F1), F01 003. (doi:10.1029/2004JF000202)

Walker, R., Dupont, T., Parizek, B. \& Alley, R. 2008 Effects of basal-melting distribution on the retreat of ice-shelf grounding lines. Geophys. Res. Letts., 35, L17 503. (doi:10.1029/2008GL034947)

Weertman, J. 1974 Stability of the junction of an ice sheet and an ice shelf. J. Glaciol., 13, 3-11.

Weertman, J. 1976 Glaciology's grand unsolved problem. Nature, 260(5549), 284-286.

Wingham, D., Wallis, D. \& Shepherd, A. 2009 Spatial and temporal evolution of pine island glacier thinning, 1995-2006. Geophys. Res. Letts., 36(17), L17 501. (doi:10.1029/2009GL039126) 


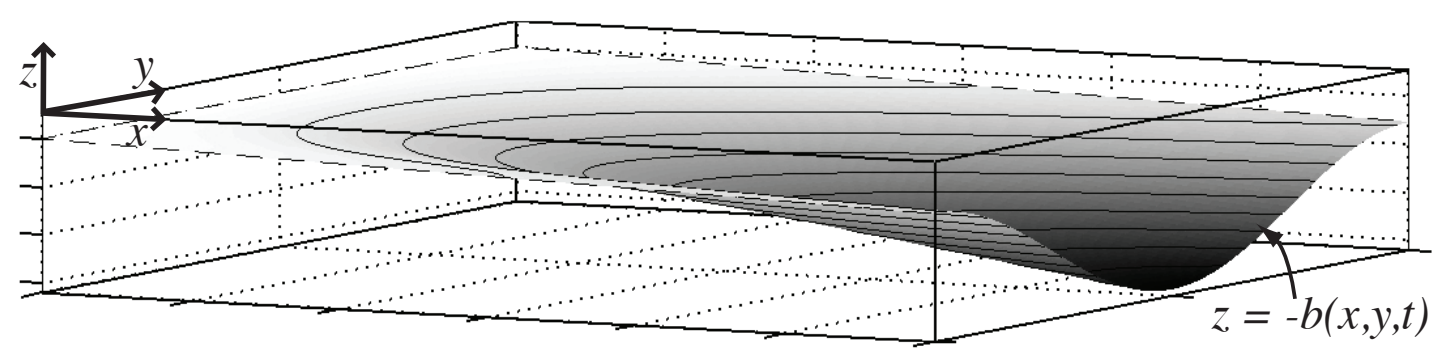

Figure 1. Schematic representation of the model domain. Periodic boundary conditions are applied at the faces of the domain with normal in the $y$-direction. The surface shown in greyscale is an example of the basal topography applied to the model. Solid black lines are depth contours; the dashed black line shows the intersection of basal surface with the edges of the domain. 

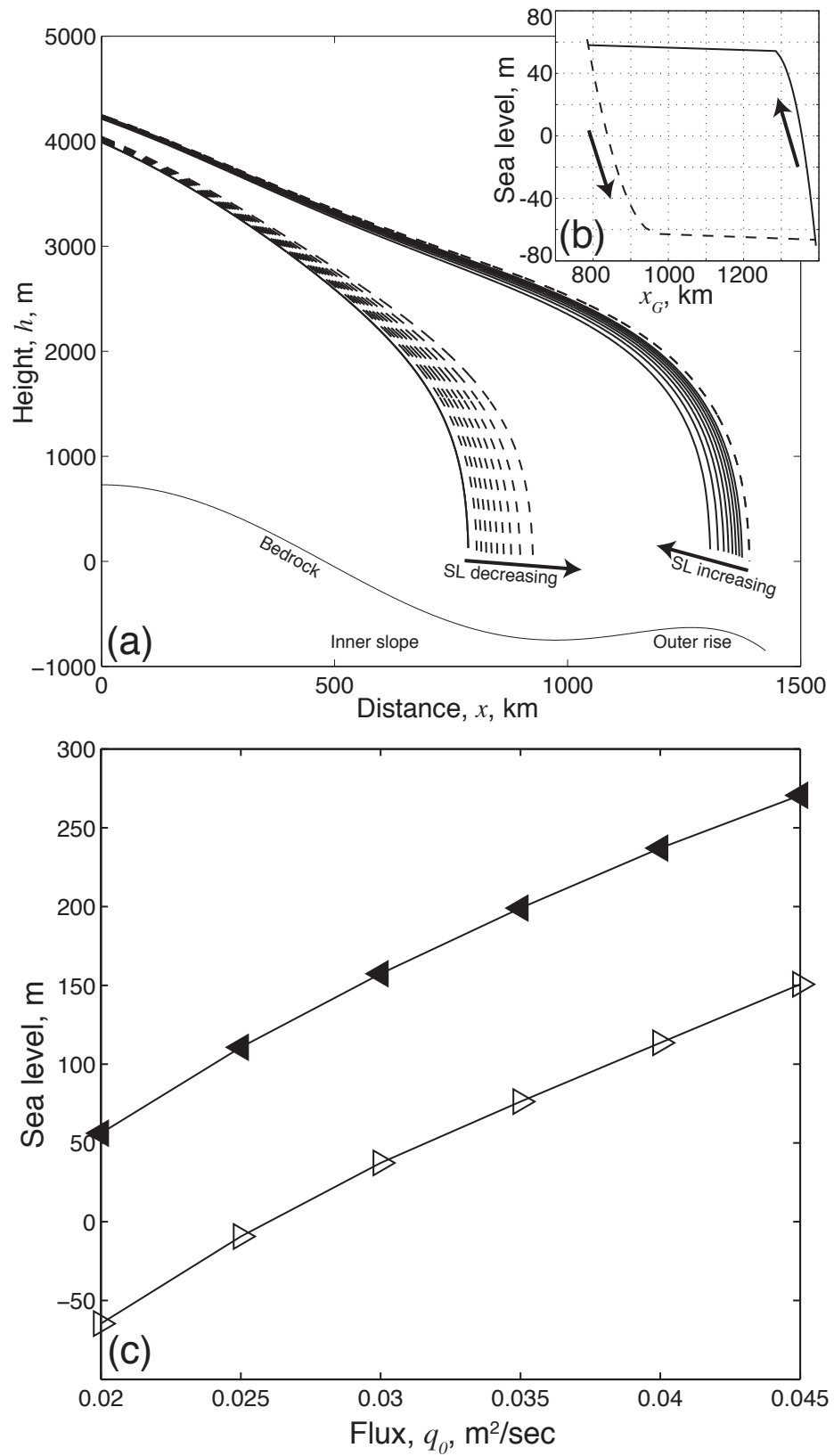

Figure 2. Hysteresis in a 2D ice-sheet model with changing sea level. Panel (a) shows steady-state curves $h(x)$ representing the height of the ice sheet in metres for different values of sea level. The dashed curves represent a series in which sea level is sinking, the solid curves a series with sea level rising. The bedrock profile is as used by Schoof $(2007 a)$. Inset panel (b) shows the hysteresis loop between the position of the grounding line $x_{G}$ and sea level. Calculation shown in (a) and (b) were performed with parameters $\mu=1 \times 10^{13}$ Pa-sec, $\rho=900 \mathrm{~kg} / \mathrm{m}^{3}, \rho_{w}=1000 \mathrm{~kg} / \mathrm{m}^{3}$, and $q_{0}=0.02 \mathrm{~m}^{2} / \mathrm{sec}$. Panel (c) shows how the hysteresis loop changes for different values of $q_{0}$. Right-pointing open triangles mark the value of sea level at which the grounding line advances; left-pointing filled triangles mark the value at which the grounding line recedes. 

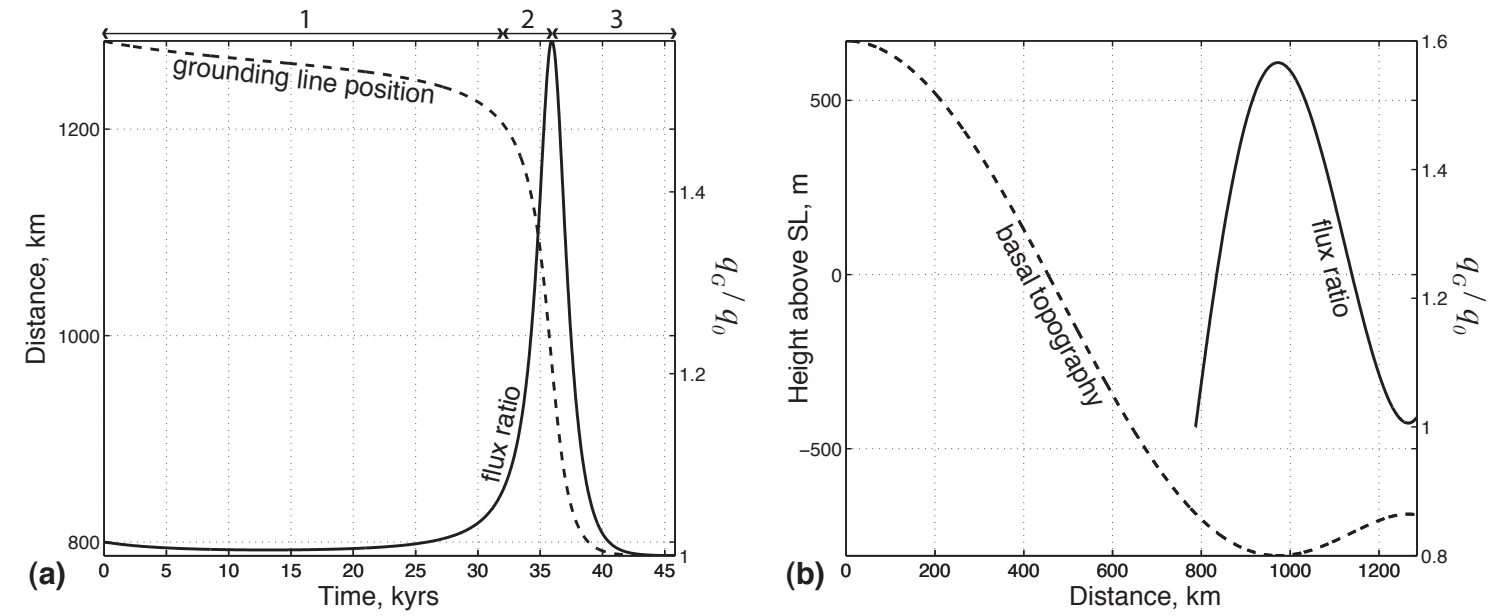

Figure 3. Ratio of grounding line flux $q_{G}$ to influx $q_{0}$ over an episode of unstable grounding line recession caused by a step-change in sea level. When $q_{G} / q_{0}=1$ the ice sheet is in steady state. Panel (a) shows the grounding line position (dashed line) and the flux ratio (solid line) as a function of time $t$ since the step-change in sea level. The three phases of the transition are delimited above the panel (see main text for explanation). Panel (b) shows the basal topography (dashed line) and the flux ratio (solid line) as a function of horizontal distance $x$. The grounding line position is initially at the right end of this plot; with time it moves to the left. The parameters for this calculation are as stated in the caption to figure 2 . The step-change in sea level was five metres. 

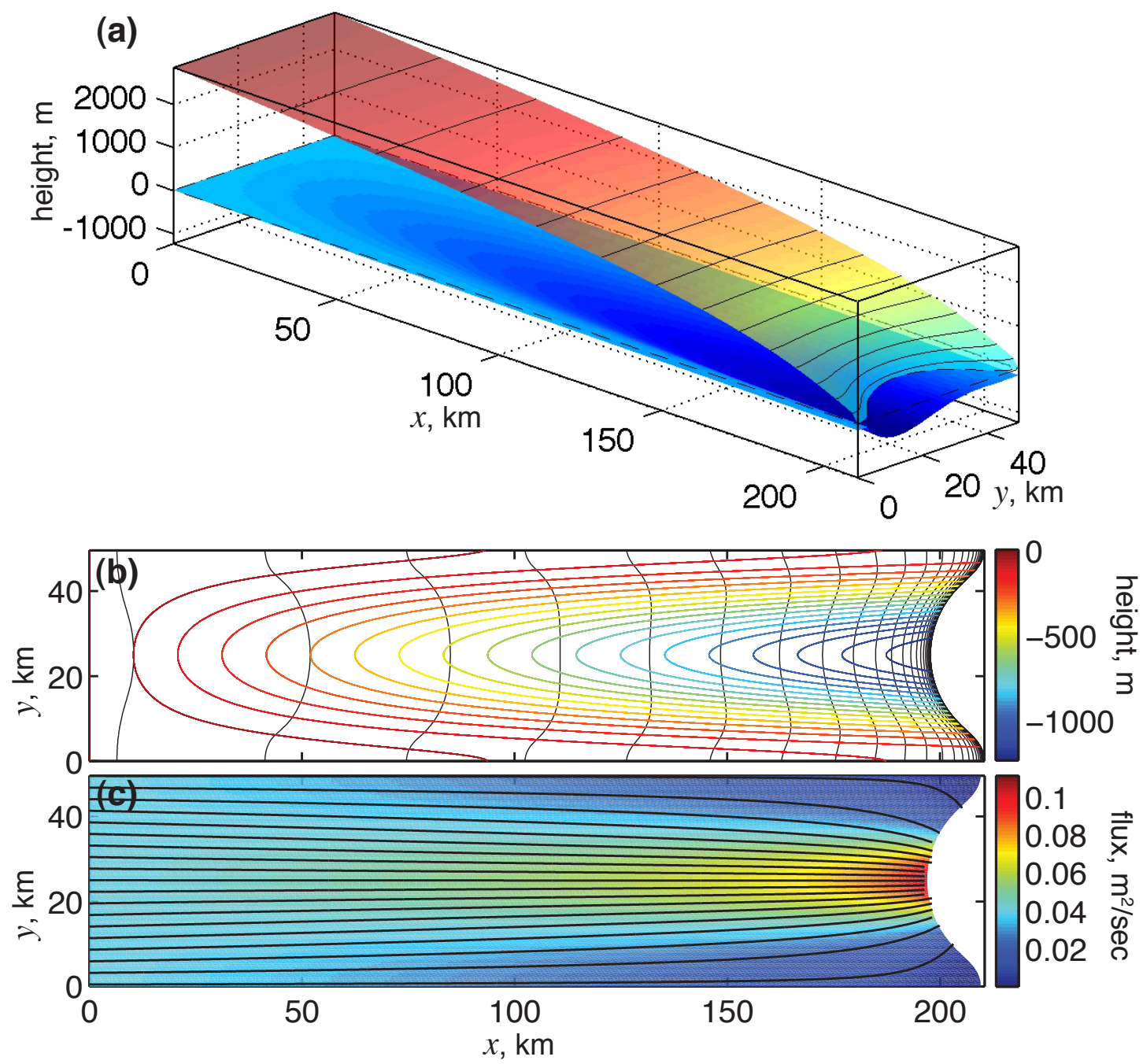

Figure 4. Results from an example calculation with variations in basal depth in the $y$-direction as described by equation (3.1) with $\alpha=3.3 \times 10^{-3}$ and $\beta=2.7 \times 10^{-3}$. Panel (a) shows the top and bottom surfaces of the ice sheet in its steady-state configuration. Sea level is at zero on the $z$-axis. The ice shelf is not shown. Panel (b) shows the grounding line position (black curves) at 50 year intervals, as it approaches steady state. Coloured curves are contours of basal depth. Panel (c) shows, in colour, the magnitude of the vertically integrated, steady-state ice flux. The black curves are streamlines. Parameters for this calculation are as follows: $\mu=1 \times 10^{13}$ Pa-sec, $\rho=900 \mathrm{~kg} / \mathrm{m}^{3}, \rho_{w}=1000 \mathrm{~kg} / \mathrm{m}^{3}$, and $q_{0}=0.04 \mathrm{~m}^{2} / \mathrm{sec}$. 


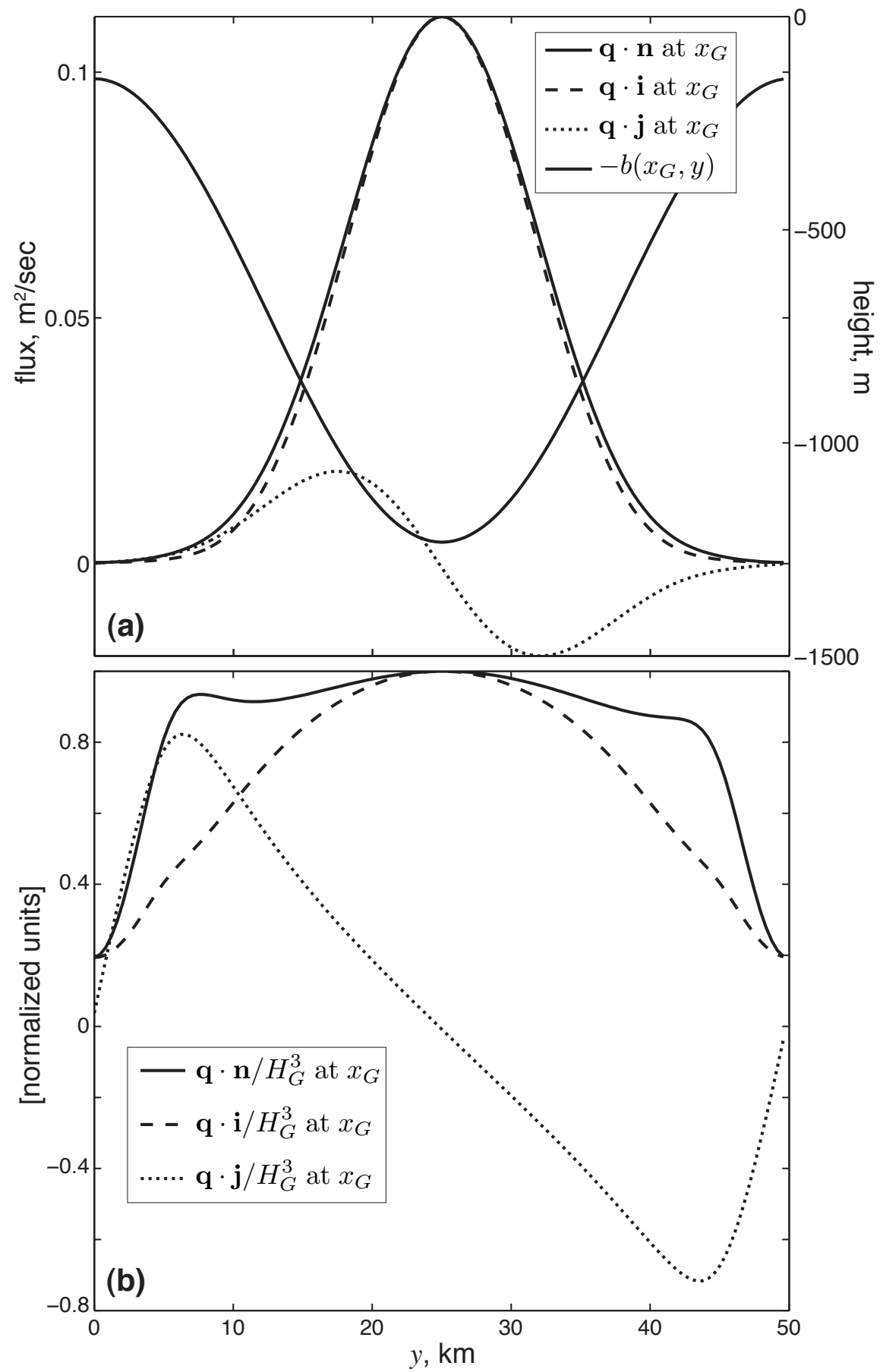

Figure 5. Characteristics of the ice flux at the grounding line, $x=x_{G}$, for the simulation shown in figure 4 $(\beta / \alpha=0.8)$. Panel (a) shows the flux normal to the grounding line, and the flux in the $x$ and $y$ directions (narrow lines, $y$-axis at left), as well as the depth of the ice-sheet base below sea level (wide line, $y$-axis at right). Panel (b) shows the flux divided by the cube of the grounding-line ice thickness $H_{G}^{3}$, and normalised by the maximum value of the $\mathbf{q} \cdot \mathbf{n}$ curve. Using equation (2.5) we can deduce that the upstream dynamics of ice flow influence the gradient in surface height at the grounding line, and thus influence the flux at the grounding line. The asymmetry in the $\mathbf{q} \cdot \mathbf{n}$ curve about $y=25 \mathrm{~km}$ is a numerical artifact. 

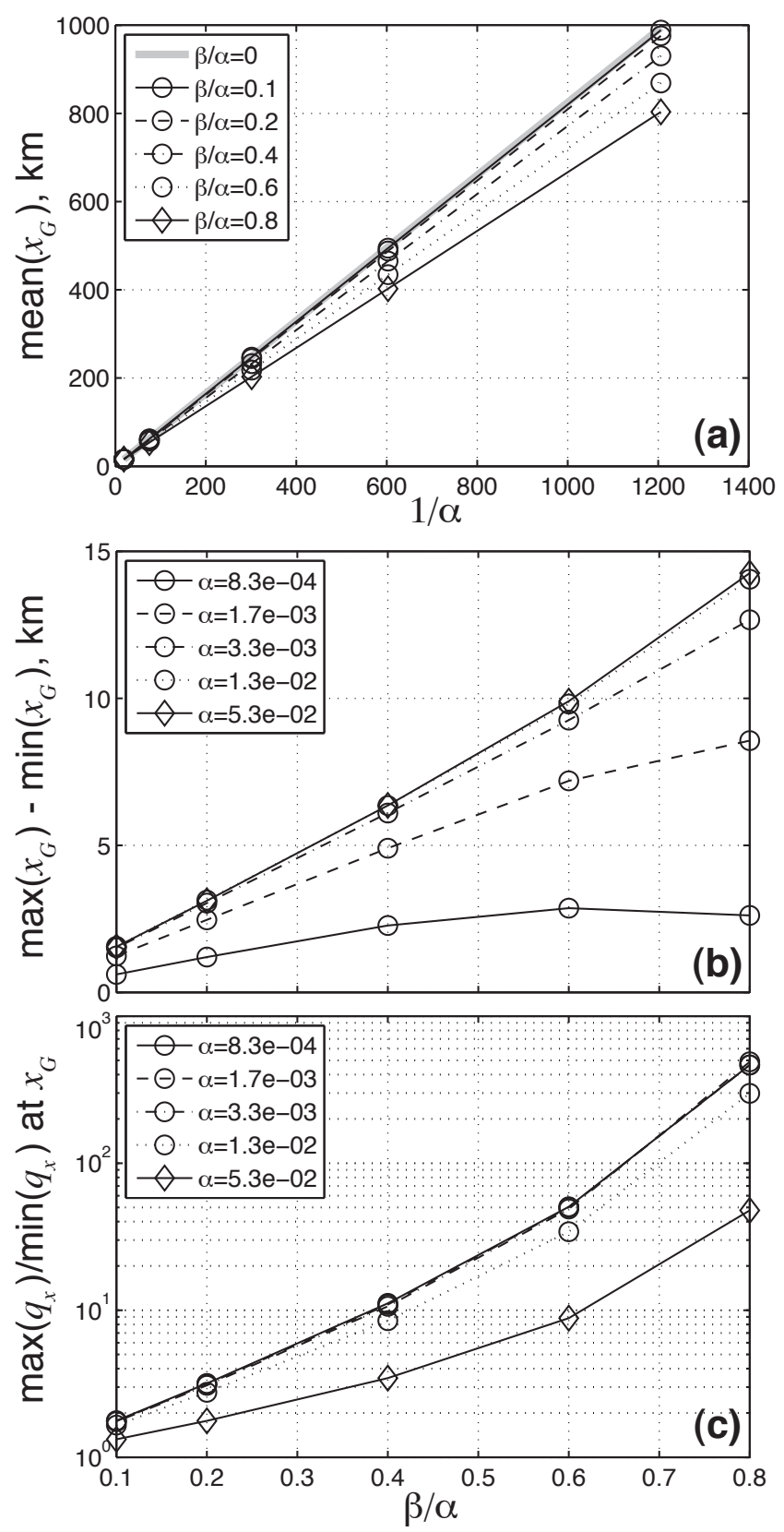

Figure 6. Variations in ice-sheet characteristics with $\alpha$ and $\beta$. Panel (a) shows the mean distance to the grounding line as a function of the inverse of the mean slope of the bed $1 / \alpha$. Steeper slopes are at the left of the diagram. Different lines represent different values of the relative perturbation in bed slope $\beta / \alpha$. The grey line is the analytical solution of Robison et al. (2009). Panel (b) shows the difference in distance between the furthest point on the grounding line and the nearest as a function of the perturbation in bed slope. A larger slope perturbation typically results in a more strongly curved grounding line. Different lines represent different values of $\alpha$. Panel (c) shows the ratio of maximum to minimum flux in the $x$-direction across the grounding line as a function of the perturbation in bed slope. The different lines are as in panel (b). In panels (b) and (c), $\alpha$ represents $\mathrm{km}$ height per $\mathrm{km} x$-distance. 


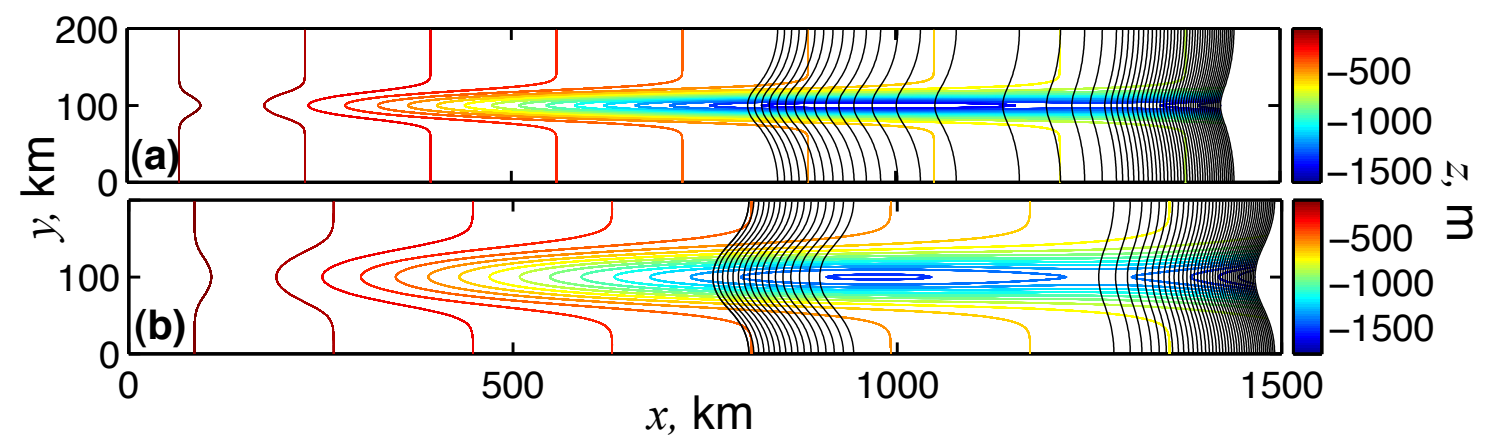

Figure 7. Grounding line positions at steady state for two different basal topography maps that differ only in their value of $\sigma$ from equation (3.2). The background slope $\alpha$ is equal to $0.52 \mathrm{~m} / \mathrm{km}$ in both cases. Each grounding line, shown in black, is a steady state solution for a different value of sea-level. Panel (a) shows a narrow central valley $(\sigma=10 \mathrm{~km})$. Sea level rises in increments of 3.2 metres. Panel (b) shows a wider central valley $(\sigma=20 \mathrm{~km})$. Sea level rises in increments of 6.3 metres. The grounding line recedes unstably in this case, while it recedes stably in panel (a). An examination of the time-dependent transition between grounding lines in (b) indicates that the difference in behaviour between (a) and (b) is not due to the difference in sea-level increments. The grid size was $102 \times 102$ in these calculations.

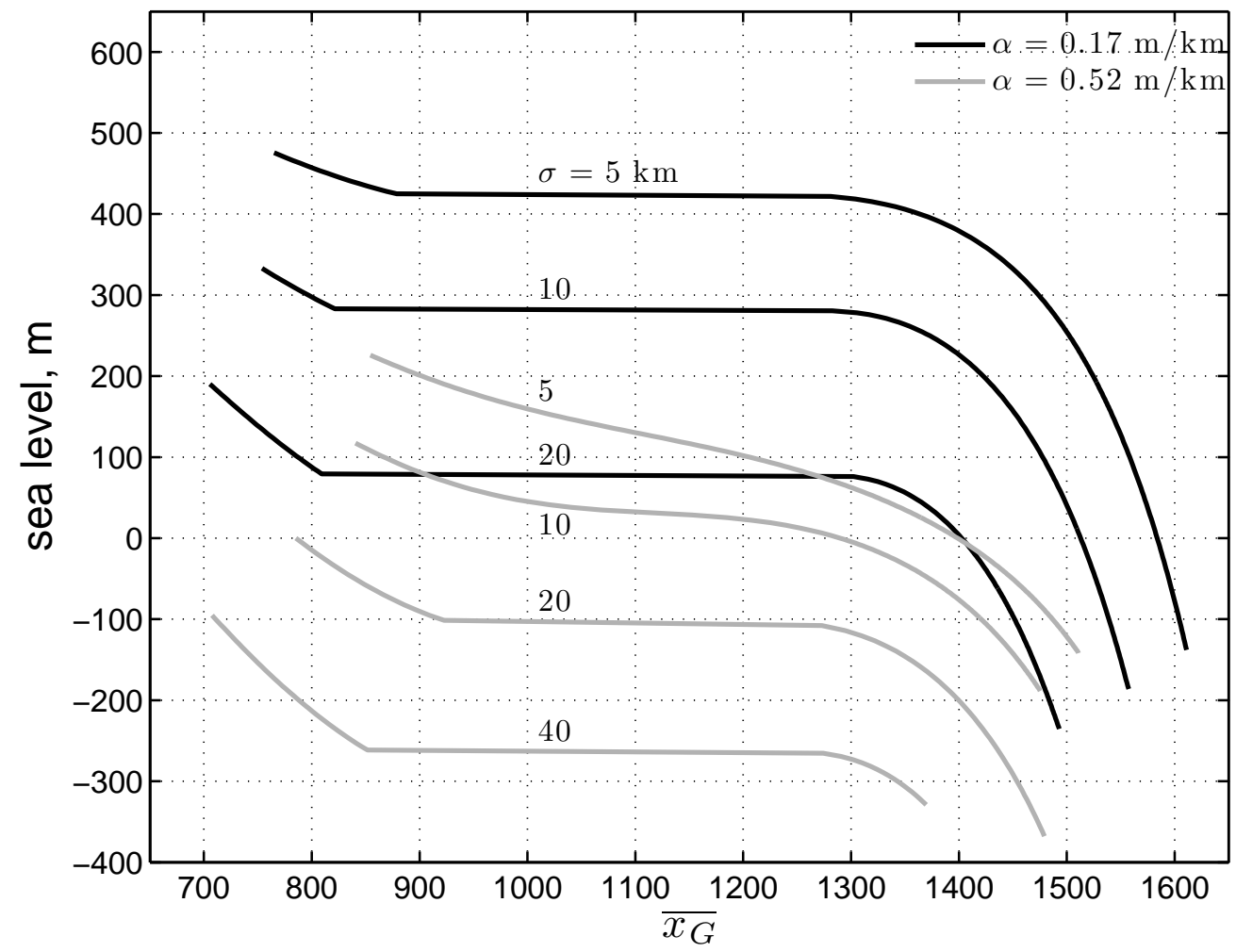

Figure 8. Summary of grounding line position (averaged in the $y$-direction) as a function of sea level for two sets of calculations with different values of $\alpha$. Mean grounding line position $\overline{x_{G}}$ is on the $x$-axis and the independent variable, sea level, is on the $y$-axis. Each curve is labelled with the corresponding value of $\sigma$ from equation (3.2). The grey curves labelled 10 and 20 are derived from the calculations shown in figure $7 \mathrm{a}$ and $\mathrm{b}$. 

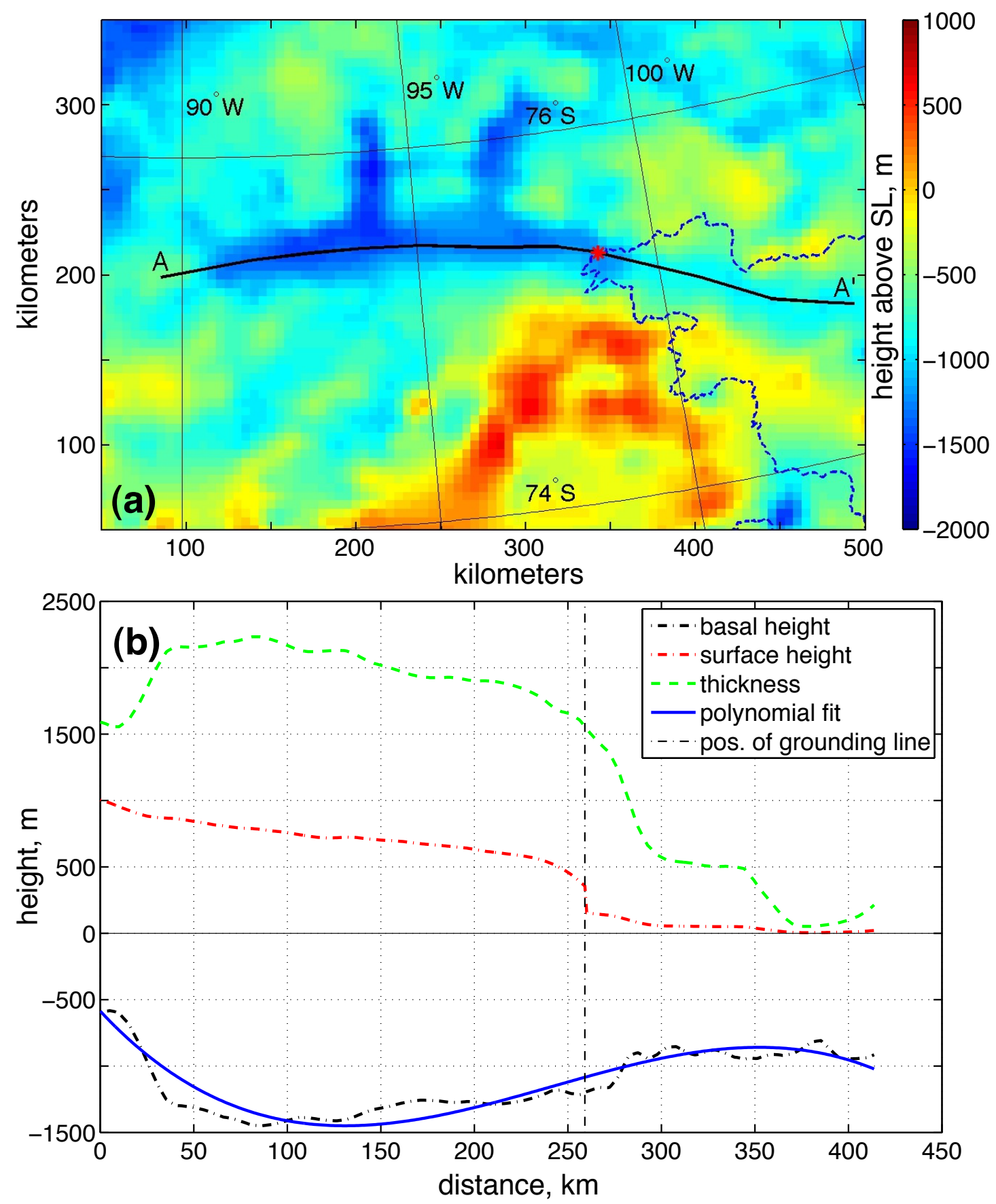

Figure 9. The Pine Island glacier as observed by the AGASEA project (Vaughan et al., 2006). Panel (a) shows a map of basal topography with respect to current sea level. The dashed line is the current grounding line from by data from the National Snow and Ice Data Center (Haran et al., 2005). The black line is a transect through the map that intersects the grounding line at the point marked with a red star. Panel (b) shows the basal topography $-b$, ice thickness $H$, and calculated surface height $h$ along the transect from A to A' in panel (a). Where the ice is grounded $h=H-b$; where the ice is floating $h=\left(\rho_{w}-\rho\right) / \rho_{w} H$ with $\rho=900$ and $\rho_{w}=1000 \mathrm{~kg} / \mathrm{m}^{3}$. The blue curve is a polynomial fit given by $y(x)=-586-15.1 x+\left(7.89 \times 10^{-2}\right) x^{2}-\left(1.01 \times 10^{-4}\right) x^{3}$ where $x$ is distance in kilometres and $y$ is height above current sea level in metres. 


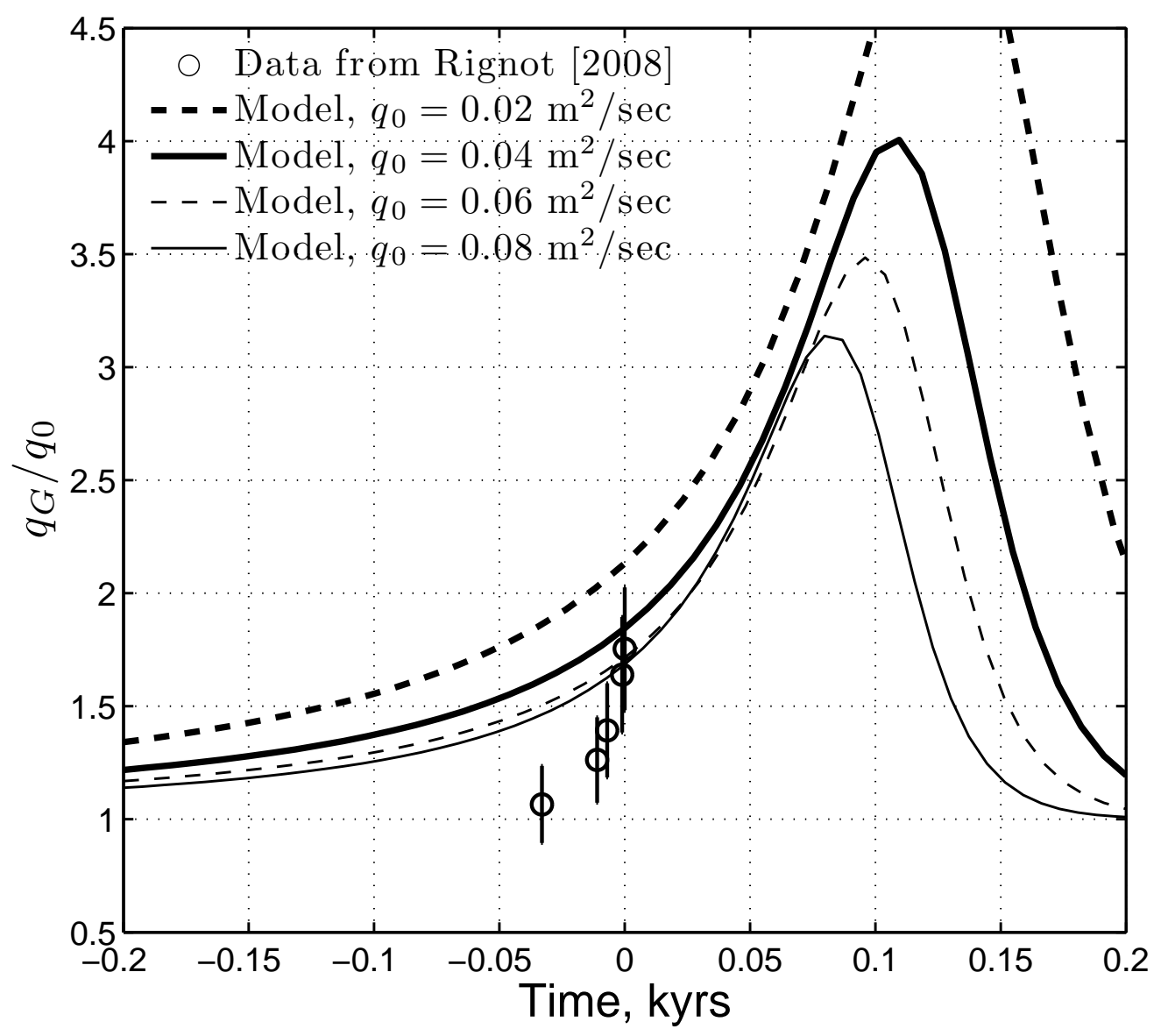

Figure 10. Flux ratio $q_{G} / q_{0}$ as a function of time. The lines represent the flux ratio calculated as in figure 3 except with basal topography as given by the blue curve in figure $9 \mathrm{~b}$, for four different values of $q_{0}$. Data points are derived from the ratio of glacial outflow to mean accumulation rate of the Pine Island glacier, as reported in table 1 of Rignot (2008). Error bars represent uncertainty propagation from the data in Rignot (2008). The curves and data are shifted in time such that the grounding line position $x_{G} \approx 260 \mathrm{~km}$ (fig. 9b) occurs at the present time $t=0$. 